\title{
The method of determining the number of excited efferent cardiac fibers in the vagus nerve of a cat
}

\begin{abstract}
Objective: Developing method of determination the number of excited efferent cardiac fibers in the cervical region of the vagus nerve of a cat by estimating the area of the glow in the high-frequency electromagnetic field inside the nerve.

Materials and methods: The observations were carried out on cats in conditions of superficial (27 cats) or deep anesthesia (13 cats out of 27) and irritation of the vagus nerve with electrical impulses (14 cats out of 27). The right vagus nerve on cat's neck was allocated and cut at the level of the lower edge of thyroid cartilage. The central end was inserted into a glass tube. Before the cut end in the transverse plane, a scanner from the unit, which creates a high-frequency electromagnetic field was installed. The glow from the scanner were recorded by a video camera through the microscope. At the same time, the ECG was recorded. Based on the ECG signal, for the processing we took the centers of glow related to the heart rhythm. The results were processed in the "STATISTIKA 6.0" program.
\end{abstract}

Results: In superficial anesthesia, the number of excitable efferent fibers was 350 or $70.0 \%$ of the available ones. In case of deep anesthesia, 193 or $38.6 \%$ accordingly. In case of nerve stimulation above the transection site all available efferent fibers have been excitable.

Conclusions: Results of this work suggest a way to identify and determine the number of excitable efferent cardiac fibers in the vagus nerve and their localization on the cross section of the central end of the nerve from the area of luminescence in high-frequency electric field.

Keywords: cardiac fibers, vagus nerve, luminescence, image processing
Volume 6 Issue 5 - 2019

\author{
Minenko IA,' Pokrovsky VM,' Tarasov \\ DG, ${ }^{2}$ Nechepurenko AA, ${ }^{2}$ Pokhotko AG,' \\ Korotkov KG, ${ }^{3}$ Ardelian AN,' Zabolotskikh \\ NV' Abushkevic VG,' Galenko-Yarochevsky \\ $\mathrm{PA}^{\prime}$ \\ 'Kuban State Medical University, Russia \\ ${ }^{2} \mathrm{FSBI}$ "Federal Center for Cardiovascular Surgery" of the \\ Ministry of Health of the Russian Federation, Russia \\ ${ }^{3}$ Federal State Budget Institution "Saint-Petersburg Scientific- \\ Research Institute for Physical Culture, Russia
}

Correspondence: KG Korotkov, Federal State Budget Institution "Saint-Petersburg Scientific-Research Institute for Physical Culture, Russia, Email korotkov2000@gmail.com

Received: August 18,2019 | Published: September 06, 2019

\section{Introduction}

To the study of efferent cardiac fibers in the vagus nerve after the classical work of Agostoni E et al., ${ }^{1}$ was devoted quite a few publications discussing the role of efferent cardiac fibers of the vagus nerve in the concept of central rhythmogenesis of the heart ${ }^{2}$ and the use of vagus nerve stimulation in cardiology. ${ }^{3}$ Registration of efferent cardiac activity in the vagus nerve, in the most accessible - cervical zone - in classical neuro-graphy is impossible because of the small number of these fibers (500 fibers out of 30,000 or $1.7 \%$ ). ${ }^{1}$ It can be detected by computer signal acquisition methods. Thus, A.G. Pohotko ${ }^{4}$ used for this purpose computer analysis by F 37 complex, which worked in the mode of separation of periodically repeating signals hidden by additive interference. He was able to identify the efferent premise to the heart in the vagus nerve in vivo and the presence of volley activity, timed to the phases of the heart cycle, at the central end of the vagus nerve in its initial state.

However, computerized mathematical processing of neural impulses at a given time interval with the subsequent "accumulation" of the useful signal requires repeated impulses, i.e. "hard" rhythm. In nature, the rhythm is not "hard", but has variability. This limits the application of this method. Another approach is the use of brush electrodes. In these electrodes of $30 \mu \mathrm{m}$ in diameter the length of the microneedles varies linearly from 0.5 to $1.5 \mathrm{~mm}$. The distance between the electrodes is $200 \mu \mathrm{m}$. Due to different electrode lengths, nerve signals can be recorded from fibers at different nerve depths. ${ }^{5,6}$
However, the likelihood of electrodes getting into the nerve bundles is small. Moreover, it is impossible to separate efferent and afferent nerve fibers. A fundamentally different method is the use of fiberoptic interfaces. The advantages of this method include the fact that the number of optoaxons will gradually approach the number of real nerve fibers. The disadvantages of the method are the relative complexity of production and the additional errors in signal conversion. ${ }^{7,8}$

Interesting studies on the registration of the excitation process in the efferent fibers of the transected vagus nerve of cats in the form of longitudinal registration of luminous foci in the high-frequency electromagnetic field were conducted by AN Ardelian. ${ }^{5}$ However, the registration of the excitation process in this study did not allow to determine the number of excitable efferent cardiac fibers in the cervical region of the vagus nerve. The aim of our work was developing method of determination the number of excited efferent cardiac fibers in the cervical region of the vagus nerve of a cat by estimating the area of the glow in the high-frequency electromagnetic field in the transverse plane inside the nerve.

\section{Materials and methods}

This study was approved at a meeting of the ethical Committee of the Kuban State Medical University. The authors of the study confirm that they have taken all measures to minimize the pain and suffering of animals in accordance with the main provisions of the European Convention for the Protection of Vertebrate Animals used 
for Experimental and Other Scientific Purposes (Strasbourg, 18 March 1986).

In the experiment 27 outbred adult cats from a University nursery were used, of both sexes, weighing 2.5-3.2 kg. All the animals were on a normal diet until the surgery. Induction into anesthesia was performed by intraperitoneal administration of sodium thiopental solution at a dosage of $40 \mathrm{mg} / \mathrm{kg}$. The right vagus nerve was isolated from the vascular nerve beam on the neck. It was cut across the lower edge of the thyroid cartilage. The central end of the transected nerve was placed in a stem tube with a diameter commensurate with that of the nerve. Opposite the cut end the scanner was installed KELSI device (ELSIS Co, St. Petersburg), which generated a high-frequency electric field, and a video camera which captured video clip through a microscope.

The excitation process at the central end of the transected vagus nerve, coming from the brain, was recorded as spreading foci of glow.

Simultaneously the ECG with cardiograph of Neurosoft Co, Ivanovo, in the form of video file was registered. Glow centers have been synchronized with ECG. Frequency filters were used to prevent the registration of the nerve excitation process lesions unrelated to cardiac activity. Moments with respiratory activity were not taken for processing. 13 cats were induced in deeper anesthesia by introducing an additional dose of sodium thiopental and similar registration was conducted.

In 14 cats irritation of the peripheral end of the transected vagus nerve with trains of electrical impulses from the ESA-1 electrostimulator formed the vagus-heart synchronization (every train consisted of 8 impulses by $2 \mathrm{~ms}$ duration and $1.5-2.0 \mathrm{~V}$ amplitude; the impulse frequency in the train was $20 \mathrm{~Hz}, 130$ trains per minute). When irritation of the peripheral nerve end was stopped, stimulating electrodes were placed under the central nerve end at the level of the upper edge of the thyroid cartilage, and after that the recording was done. Thus, the registration of the excitation process from the transverse surface of the transected vagus nerve of a cat was carried out with superficial anesthesia, with deep anesthesia and after the stimulation of the central end of the vagus nerve by train of electrical impulses above the transection zone. Statistika 6.0 program was used to determine the normality of distribution. Normal distribution allowed using parametric methods of statistical processing, in particular, Student's t-criterion. Reliable difference between means was considered at $\mathrm{p}<0.05$

\section{Results}

In the high-frequency electromagnetic field, at the central end of the transected vagus nerve, in the transverse plane, the excitation process associated with the ECG cycle was manifested against the background of neural "noise" in the form of small foci of glow (Figure 1 ), and in the form of two large foci of glow, which are marked as proximal (Figure 2) and distal (Figure 3) to the heart plane. Parameters of the glow foci in the nerve depths during superficial anesthesia, deep anesthesia and irritation by electrical impulses of the nerve above the place of transection are presented in the Table 1.

Foci of the glow were localized in the nerve depth in one sector. They were located nearby, but not on the same axis. From this follows that the process of excitation from the brain to heart spread out along different, closely located bundles of nerve fibers. The area of the proximal focus of the glow is less than the area of the distal focus in surface anesthesia by $35.8 \%$, in deep anesthesia by $78.2 \%$, and in case of irritation by electrical impulses by $75 \%$. Since the area of the focus depends on the number of excited nerve fibers, we may assume that in case of anesthesia a greater number of fibers, which excitation forms a proximal focus of glow is suppressed. In the conditions of nerve stimulation by electrical impulses the response of functioning fibers that cause a distal luminescence is bigger than the fibers of the proximal luminescence.

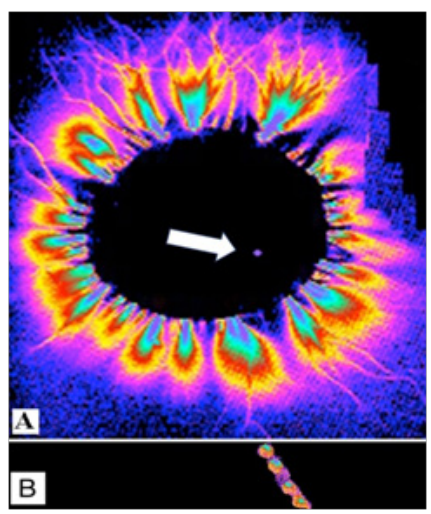

Figure I Cat I: Recording of the glow from the central end of the transected vagus nerve in the high-frequency electromagnetic field. $A$ - in the transverse plane and B - longitudinally. Focal area of glow is $0.04 \%$ of the nerve crosssection area $(6 \times 3 \mathrm{~mm})$.

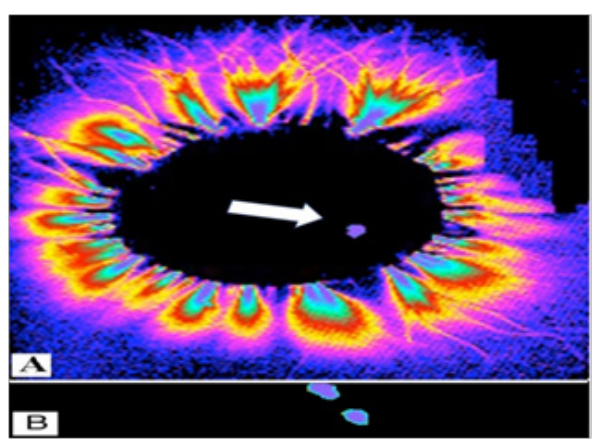

Figure 2 Cat I: Recording of the glow from the central end of the transected vagus nerve in the high-frequency electromagnetic field. $A$ - in the transverse plane and $B$ - longitudinally, when the proximal focus (signal) passes from the brain. Focal area of glow is $0.04 \%$ of the nerve cross-section area $(6 \times 3 \mathrm{~mm})$.

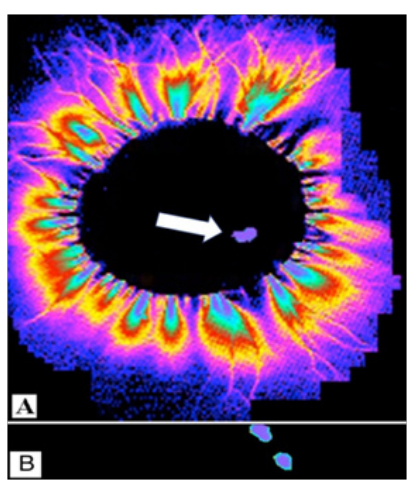

Figure 3 Cat I. Recording of the glow from the central end of the transected vagus nerve in the high-frequency electromagnetic field. $A$ - in the transverse plane and $B$ - longitudinally, when the distal focus (signal) passes from the brain. Focal area of glow is $0.04 \%$ of the nerve cross-section area $(6 \times 3 \mathrm{~mm})$. 
Table I Parameters of the vagus nerve in the cervical area and glow foci in the nerve lining reflecting the excitation process

\begin{tabular}{|c|c|c|c|c|c|c|c|c|c|}
\hline & \multicolumn{3}{|c|}{ Superficial anesthesia } & \multicolumn{3}{|c|}{ Deep anesthesia } & \multicolumn{3}{|c|}{ Electrical impulses } \\
\hline \multicolumn{10}{|c|}{ Nerve area SC on the scan, $\mathbf{m m}^{2}$} \\
\hline $\mathrm{n}$ & \multicolumn{3}{|l|}{27} & \multicolumn{3}{|l|}{13} & \multicolumn{3}{|l|}{14} \\
\hline M & \multicolumn{3}{|l|}{8476,3} & \multicolumn{3}{|l|}{8656,6} & \multicolumn{3}{|l|}{8596,4} \\
\hline SD & \multicolumn{3}{|l|}{66,5} & \multicolumn{3}{|l|}{37,6} & \multicolumn{3}{|l|}{50,1} \\
\hline \multirow[t]{3}{*}{$\pm \mathrm{m}$} & \multicolumn{3}{|l|}{12,8} & \multicolumn{3}{|l|}{10,4} & \multicolumn{3}{|l|}{13,4} \\
\hline & \multirow{2}{*}{$\begin{array}{l}\text { Back } \\
\text { ground }\end{array}$} & \multicolumn{2}{|c|}{ Glow foci } & \multirow{2}{*}{$\begin{array}{l}\text { Back } \\
\text { ground }\end{array}$} & \multicolumn{2}{|c|}{ Glow foci } & \multirow{2}{*}{$\begin{array}{l}\text { Back } \\
\text { ground }\end{array}$} & \multicolumn{2}{|c|}{ Glow foci } \\
\hline & & $P$ & d & & $\mathrm{P}$ & $d$ & & $\mathrm{P}$ & d \\
\hline \multicolumn{10}{|c|}{ Glow area, $\mathrm{mm}^{2}$} \\
\hline $\mathrm{n}$ & 27 & 27 & 27 & 13 & 13 & 13 & 14 & 14 & 14 \\
\hline M & 3,0 & 74,2 & 100,0 & 3,2 & 12,0 & 55,1 & 3,3 & 50,0 & 200,0 \\
\hline SD & 0,5 & 9,8 & 12,0 & 0,4 & 0,7 & 11,5 & 0,4 & 7,8 & 14,2 \\
\hline$\pm \mathrm{m}$ & 0,1 & 1,9 & 2,3 & 0,1 & 0,2 & 3,2 & 0,1 & 2,1 & 3,8 \\
\hline \multicolumn{10}{|c|}{ Amount of fibers, thousands } \\
\hline $\mathrm{n}$ & 27 & 27 & 27 & 13 & 13 & 13 & 14 & 14 & 14 \\
\hline M & 11 & 74 & 350 & 10 & 42 & 193 & 12 & 175 & 700 \\
\hline SD & 0,4 & 10,4 & 21,8 & 0,7 & 5,0 & 14,4 & 0,8 & 13,0 & 19,1 \\
\hline$\pm \mathrm{m}$ & 0,2 & 2,0 & 4,2 & 0,2 & $\mathrm{I}, 4$ & 4,0 & 0,2 & 3,5 & $5, I$ \\
\hline \multicolumn{10}{|c|}{$\%$ from cordial fibers. Amount of cordial fibers about $30,000^{\prime}$} \\
\hline & 0,4 & 2,5 & 11,7 & 0,3 & $\mathrm{I}, 4$ & 6,4 & 0,4 & 5,8 & 23,3 \\
\hline \multicolumn{10}{|c|}{$\% \%$ from cordial efferent fibers. Amount of efferent cordial fibers about $500^{\prime}$} \\
\hline & 2,2 & 14,8 & 70,0 & 2,0 & 8,4 & 38,6 & 2,4 & 35,0 & 140,0 \\
\hline
\end{tabular}

$\mathrm{N}$, amount of animals; $M$, mean arithmetic; SD, mean square deviation; $\pm m$, mean arithmetic error; $p$, proximal luminescence; $d$, distal luminescence.

According to Agostoni E et al., ${ }^{1}$ there are about 30,000 nerve fibers in the cervical vagus nerve. If we assume that most of the fibers have the same diameter, the cross-sectional area on the histogram is about $8476 \mathrm{~mm}^{2}$, and $1 \mathrm{~mm}^{2}$ corresponds to 3.5 fibers (30000/8476). According to this ratio, it is possible to obtain the number of efferent cordial fibers involved in the process of excitation from the area of the glow in the depths of the nerves. From the above materials follows that the number of excitable efferent fibers in surface anesthesia is greater than in deep anesthesia. When nerves are irritated by electrical impulses, it excites not only all efferent cordial fibers, but non-efferent fibers as well. Therefore, the model of vaginal-heart synchronization does not fully reflect the processes of excitation of efferent cordial fibers occurring in vivo.

\section{Conclusions}

Results of this work suggest a way to identify and determine the number of excitable efferent cardiac fibers in the vagus nerve and their localization on the cross section of the central end of the nerve from the area of luminescence in high-frequency electric field.

\section{Acknowledgements}

None.

\section{Conflicts of interest}

The authors confirm that this article content has no conflicts of interest.

\section{Funding details}

None.

\section{References}

1. Agostoni E, Chinnock JE, De Burgh DM, et al. Functional and histological studies of the vagus nerve and its branches to the heart, lungs and abdominal viscera in the cat. $J$ physiol. 1957;135(1):182-205.

2. Pokrovskiy VM. Formation of heart rhythm in human and animal organisms. Krasnodar: Kuban-book; 2007.

3. Syrovnev VA, Lebedev DS, Mikhailov EN. Vagus nerve stimulation in cardiology. Translational medicine. 2017;4(2):6-16.

4. Pohotko AG. Analysis of efferent signals in the vagus nerve. Kuban scientific medical journal. 2000;50(2):38-40.

5. Ardelian AN. Identification of efferent and afferent activity, timed to the heart rhythm in the vagus nerve of a cat. Kuban scientific medical journal. 2015;153(4):13-17.

6. Safin DR, Pilshchikov IS. Application of the implantable microelectrodes in the prosthetic control systems. Electronics, measuring equipment, radio engineering and communication. 2010;14(2):104-109.

7. Nikitin VS. Multichannel Fiber Optic Neuro Interfaces. Nanoindustry. 2009;1:30-34.

8. Schwartz AB, Cui XT, Weber DJ, et al. Brain-controlled interfaces: movement restoration with neural prosthetics. Neuron. 2006;52:205220 . 